\title{
The influence of system settings on positioning accuracy in acoustic telemetry, using the YAPS algorithm
}

\author{
Jenna Vergeynst ${ }^{1 *+}$ (D) , Henrik Baktoft ${ }^{2 \dagger}$, Ans Mouton $^{3}$, Tom De Mulder $^{4}$, Ingmar Nopens $^{1}$ and Ine Pauwels ${ }^{3}$
}

\begin{abstract}
Background: Acoustic positioning telemetry allows to collect large amounts of data on the movement of aquatic animals by use of autonomous receiver stations. Essential in this process is the conversion from raw signal detections to reliable positions. A new advancement in the domain is Yet Another Positioning Solver (YAPS), which combines the detection data on the receivers with a model of animal movement. This transparent, flexible and on-line available positioning algorithm overcomes problems related to traditional point-by-point positioning and filtering techniques. However, its performance has only been tested on data from one telemetry system, providing transmitters with stable burst interval. To investigate the performance of YAPS on different system parameters and settings, we conducted a simulation study.

Results: This paper discusses the effect of varying burst types, burst intervals, number of observations, reflectivity levels of the environment, levels of out-of-array positioning and temporal receiver resolution on positioning accuracy. We found that a receiver resolution better than $1 \mathrm{~ms}$ is required for accurate fine-scale positioning. The positioning accuracy of YAPS increases with decreasing burst intervals, especially when the number of observations is low, when reflectivity is high or when information out-of-array is used. However, when the burst interval is stable, large burst intervals (in the order of 1 to $2 \mathrm{~min}$ ) can be chosen without strongly hampering the accuracy (although this results in information loss). With random burst intervals, the accuracy can be much improved if the random sequence is known.

Conclusions: As it turns out, the key to accurate positioning is the burst type. If a stable burst interval is not possible, the availability of the random sequence improves the positioning of random burst interval data significantly.
\end{abstract}

Keywords: YAPS, Acoustic positioning telemetry, Simulation study, Sensitivity analysis, Fine-scale positioning

\section{Background}

The study of aquatic animal behaviour has advanced largely with the development of radio and acoustic telemetry in the late 1950 s (e.g. [1-4]). These underwater positioning techniques allow collecting large amounts of data on the movement of individual animals [5]. Since radio

\footnotetext{
*Correspondence: Jenna.Vergeynst@UGent.be; jennavergeynst@gmail.com †Jenna Vergeynst and Henrik Baktoft contributed equally to the manuscript

${ }^{1}$ Department of Data Analysis and Mathematical Modelling, Ghent University, Coupure Links 653, 9000 Ghent, Belgium

Full list of author information is available at the end of the article
}

waves do not propagate efficiently in saltwater and the technology is not yet suitable for fine-scale $2 \mathrm{D}$ positioning $[6,7]$, the most popular technique is acoustic telemetry [8]. This allows tracking of, for instance, diadromous fish species, which spent one part of their life in freshwater, and the other part in the sea (e.g. [5, 9-12]). Acoustic positioning telemetry systems consist of stand-alone receivers fixed below the water surface. The animals of interest are tagged with acoustic transmitters, producing a signal every second or minute (or other time interval, depending on the research question). The unique ID of the animal is encoded in the signal, which can be 
decoded by the receivers or at the post-processing phase. The independence of the system enables researchers to collect huge datasets with minor data collection effort, once the set-up of the system is established. However, the main task starts once the data collection is done: converting the raw detections into reliable animal positions.

Most manufacturers provide a (paid) service or software to aid their customers in this process (e.g. Vemco, Lotek, Thelma Biotel). They calculate the positions using the Time Difference Of Arrival (TDOA) algorithm, a hyperbolic positioning technique. When the signal is detected by two receivers, the differences in arrival time form a hyperbola of possible positions [13]. Detection on a third receiver is needed to provide a second hyperbola, and its intersection with the first hyperbola determines the position in 2D. (In reality, these systems work in 3D, where three receivers determine a set of hyperboloids and the (measured or assumed) depth of the transmitter determines the horizontal plane on which the transmitter position can be found [13]). This is a point-by-point method: every position is calculated independently of all other transmissions. If one receiver picks up an erroneous signal, the shift in the resulting hyperbola might completely dislocate the intersection point with other hyperbolas, causing a positioning error of up to several hundreds of meters. Usually, the positions come with an error indication that the customer can use to filter the data. However, these filters cannot cope with unpredictable phenomena such as reflections (also called multipath) of the acoustic signal against hard surfaces, e.g. rock formations and concrete walls ([14]; communication with manufacturers).

A recent advancement in the domain of acoustic positioning is Yet Another Positioning Solver (YAPS; [15]). In contrast to the TDOA algorithm, YAPS directly uses the time of arrival (TOA) data on all receivers and estimates a track by fitting a model of animal movement on these data. In an iterative process, YAPS simultaneously estimates the time of transmission, speed of sound (if no measurements available), and $X$ and $Y$ coordinate of all positions. The algorithm was originally developed for data obtained using equipment from one specific manufacturer (Lotek Wireless Inc., Newmarket, Ontario, Canada), with stable burst intervals. It has since been extended to random burst interval data, which are used in transmitters from other manufacturers (e.g. Thelma Biotel, Trondheim, Norway and Vemco, Halifax, Canada). However, its performance on random burst interval data and other system characteristics has not been rigorously tested.

Depending on the manufacturer, study site and research questions, widely different settings and system parameters might influence positioning accuracy. The extent of this influence depends on the applied positioning algorithm. The hardware determines specific settings, such as burst type and burst interval of the transmitters and temporal resolution of the receiver. The study site and set-up determine more general characteristics of the dataset, such as the number of detections per animal, origin of detections with respect to the receiver array, and the number of detections originating from reflected signals. In this study, we investigated the sensitivity of YAPS to these six parameters, which are further elaborated in the following paragraphs.

Transmitters are characterised by burst interval and burst type. The burst interval is the time between subsequent transmissions, whilst the burst type indicates the distribution of burst intervals. When each subsequent burst interval is the same, the burst type is called 'stable'. Often, the burst intervals vary randomly between two fixed limits (e.g. between 20 and 40 s). In this case, the burst type is called 'random. A random burst interval prevents that two transmitters simultaneously present in the receiver array, would ping in-phase and continuously overlap [16]. In fact, the interval is not truly random, but follows a computer-generated random sequence (i.e. pseudo-random; personal communication with Vemco and Thelma Biotel). When this sequence is known, YAPS can utilise this information to predict the time of next transmission.

The choice of burst interval is mostly a trade-off between battery life of the transmitter and desired temporal resolution of the tracks. An additional constraint to burst interval is the duration of a transmission, which depends on the type of signal encoding. A frequently used signal encoding is pulse position modulation (PPM), where the ID is encoded in the time interval between 8 and 10 transmitted pulses [17-19]. Since a PPM transmission takes 3 to $5 \mathrm{~s}$ [20], simultaneous transmissions of different animals can cause signal collision, which results in incomplete or corrupted transmissions. Hence, the number of fish that will be present simultaneously in the study area limits the allowable burst interval. In contrast, Binary Phase Shift Key (BPSK) technology allows transmitting the encoded ID and even sensor data in $<10 \mathrm{~ms}$, using phase modulation to encode information [21]. This technology is used for instance in the Vemco High Residency (HR) coded tags [20]. Another technique that allows similarly fast transmission is Code Division Multiple Access (CDMA), for instance used by Lotek [22, 23]. Both HR and CDMA allow the simultaneous presence of hundreds of animals, without severely limiting the frequency of signal transmission and hence still allowing short burst intervals.

The temporal resolution of the receiver expresses the accuracy with which a transmission's time of arrival is 
measured, and depends on the electronics of the receiver and the type of signal transmission. In the case of PPM, the signal is decoded by analogue receivers [21]. A signal is recorded once it has reached a certain amplitude at the receiver, so the exact moment of time of arrival cannot be measured very accurately. Hence, PPM has an accuracy of 1 to $2 \mathrm{~ms}$ (personal communication with Vemco). In contrast, digital technologies such as BPSK and CDMA allow to measure the time of arrival up to sub-millisecond precision. Although this parameter affects the positioning accuracy independently of the positioning algorithm (since it sets a physical limit to the possible accuracy), a simulation study allows to investigate its effect on positioning accuracy in combination with other factors. Note that a positioning system with independent receivers (i.e. not connected to each other or to a common computer clock) requires time synchronisation of the receiver clocks, which are prone to drifting [13]. However, this is not related to temporal receiver resolution, and in this study we assume that the receivers are synchronised (see $[24,25]$ for time synchronisation of real data).

The number of detections (observations) per animal depends on the interplay between burst interval and fish swimming speed. When a fish swims slowly through the array and the burst interval of its transmitter is not too large, it will be detected often and result in a large amount of data. However, when a fish swims fast through the receiver array and has a large burst interval-transmitter, very few signals might be detected on the receiver set. Point-by-point positioning methods such as TDOA can calculate a position no matter the number of transmissions, as long as the signal is detected by at least three receivers. In contrast, the YAPS algorithm needs a minimum amount of data to fit the movement model to the TOA data.

Although the main zone of interest is generally inside the array, for instance covering part of a lake, pond or marine area, or a section of a river or canal, the behaviour just before arriving at the covered section might be useful extra information. TDOA methods have difficulties in treating signals that originate from outside the receiver array contours [13, 22]; even to the extent that sometimes noisy tracks inside the array turn out to originate from out-of-array signals ([14]).

Finally, the environment of the study site determines the number of reflected detections present in the data. Acoustic signals bounce off hard surfaces, causing an extension of the acoustic path: the reflected signal arrives later at the receiver than a direct signal, adding an error to the TOA information and hence to the resulting position. Many environments contain reflective surfaces: signals can for instance bounce off the ocean floor, the water surface and rock formations [26]. Concrete structures, such as canal walls, sluices, dams and other human-made hydraulic structures, reflect even up to $98 \%$ of acoustic energy [27]. Mostly, receivers have at least some protection against reflected signals, for instance by use of blanking (i.e. making the receiver deaf for a specified period) after detection of the direct signal [26]. However, the direct signal might not be heard, or if the site is large enough (i.e. the reflecting surface is far away), the reflected signal might arrive after the blanking period.

In this study, we investigated the sensitivity of the YAPS algorithm to the six parameters or system properties discussed above: burst type, burst interval, number of observations, reflectivity of the environment, out-ofarray positioning and temporal receiver resolution. To this end, we simulated and estimated 50 tracks for all considered combinations of settings $(17,850$ tracks in total). We varied the parameters between values that we have encountered in our own experiences or in literature, or that are used by manufacturers we have worked with. This study aims to help aquatic ecologists in the choice of system settings and to inform users and manufacturers on potential areas of improvement.

\section{Results}

In the following sections, we compare the performance of YAPS on the combined effect of signal characteristics (i.e. burst type and interval) and one of the following parameters: number of observations (track length), reflectivity, out-of-array and temporal receiver resolution. Therefore, we visualise and discuss the median error (with error being the distance between simulated and estimated positions; Fig. 1) and the standard deviation on the error (Fig. 2) of each simulated track.

\section{Effect of signal characteristics}

Regardless of the value of other parameters, the performance of YAPS increases with burst type going from random over known-random to stable (Fig. 1). In general, the median error on a track also increases with burst interval, but for large stable and known random burst intervals, burst interval does not affect the error significantly (except in highly reflective environments, Fig. 1b). The standard deviation on the error increases with burst interval in all situations (Fig. 2). Remarkably, stable and known random burst interval tracks have almost the same spread of errors, whilst the spread on random burst interval tracks can be 2 to 4 times higher.

\section{Effect of number of observations}

Tracks with large mean burst interval (especially 60 and 90 s) suffer most from short track lengths, with errors that are 2 to 4 times larger for tracks of $500 \mathrm{~s}$ than for tracks of 10,000 s (Fig. 1a). However, the spread on the 


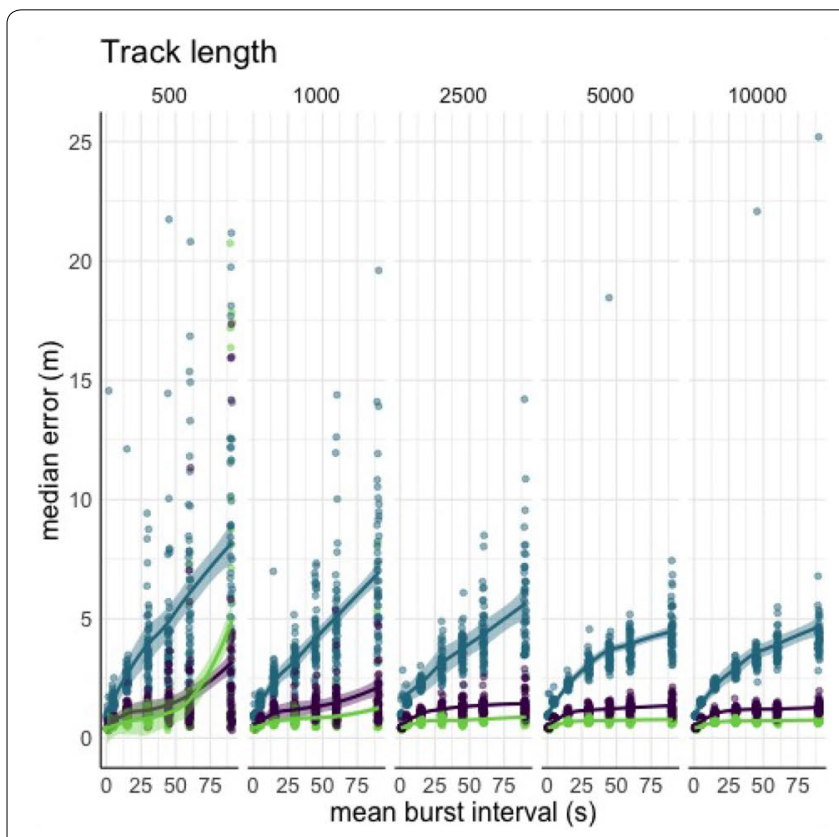

a

\section{Out-of-array shifts}

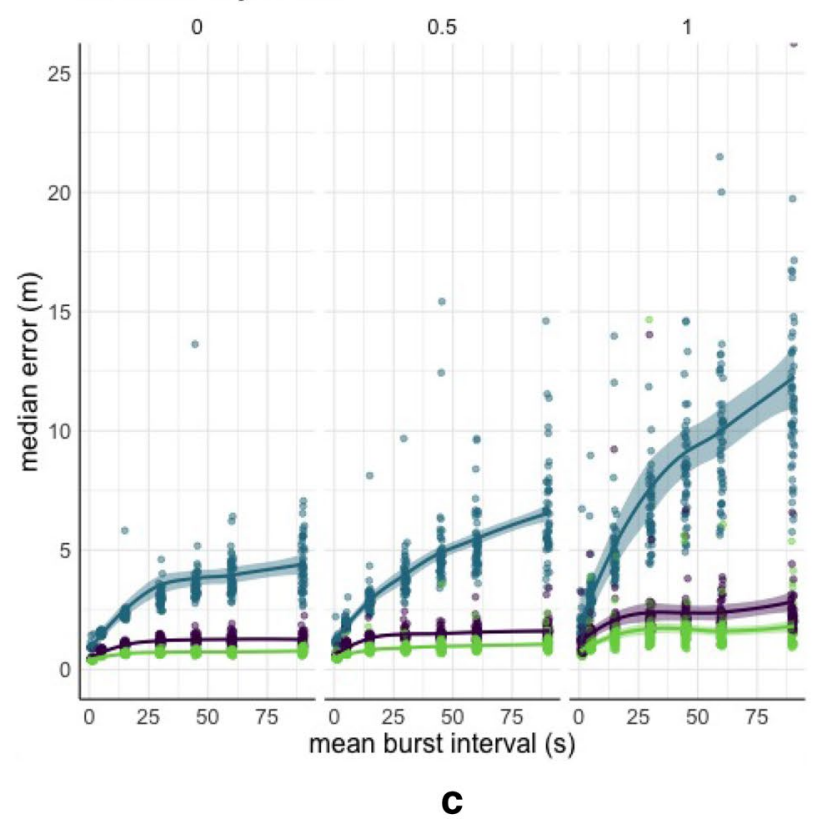

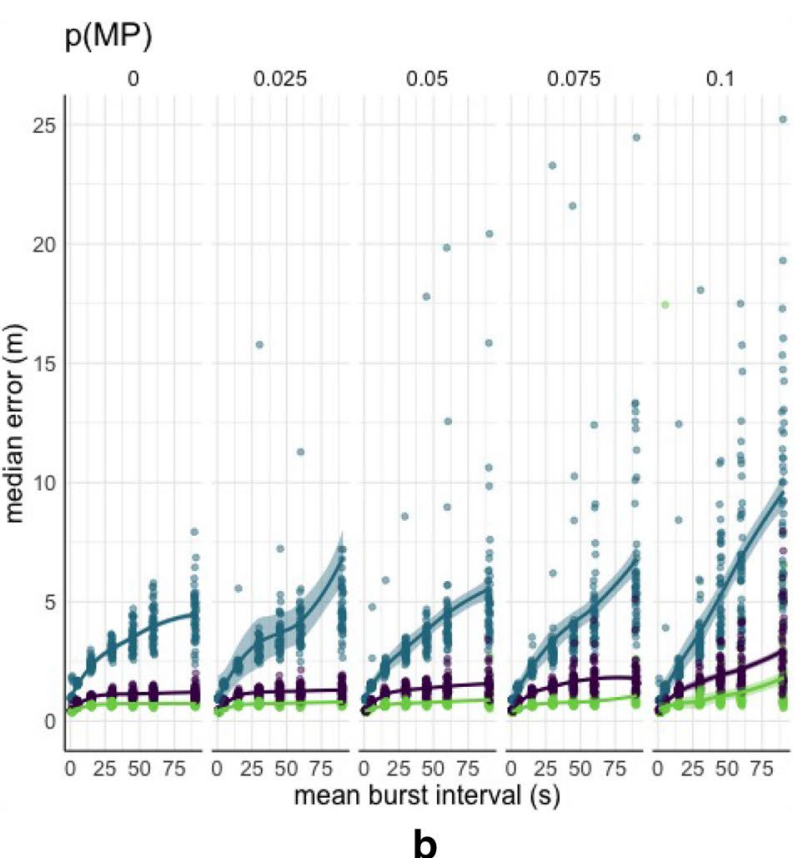

Temporal receiver resolution (1E-6 s)

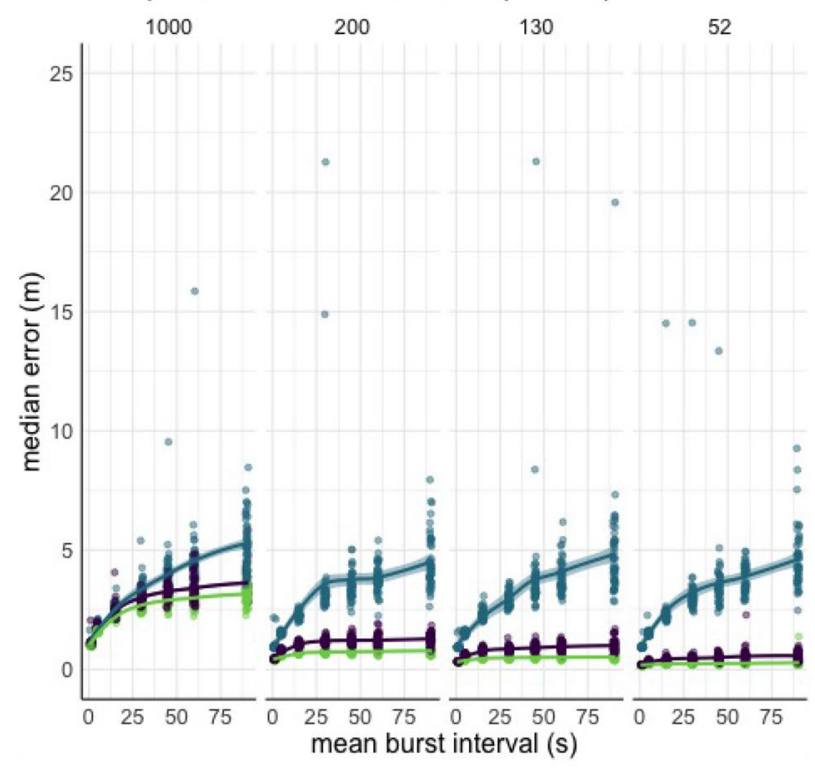

d

Fig. 1 YAPS performance in different settings. The four plots show the combined effect of burst interval properties with a varying track lengths, b probabilities of multipath $\mathrm{p}(\mathrm{MP})$, $\mathbf{c}$ shifts out-of-array and $\mathbf{d}$ temporal receiver resolution. Each panel shows the results for random burst interval (blue), known random burst interval (purple) and stable burst interval (green) and for different mean burst intervals ( $x$-axis). Each dot represents the median error of one track. Each combination of settings was run on 50 tracks. When not under variation, track length, $p(M P)$, shift and resolution were respectively fixed at 5000, 2.5\%, 0 and $200 \mu$ s. The line on top of the data is a LOcally Estimated Scatterplot Smoothing (LOESS) smoother with 95\%-confidence interval showing respectively the general pattern (i.e. average value per setting) and the uncertainty on the smoother. To enhance the visualisation, median errors above $25 \mathrm{~m}$ are not shown. The maximum occurring median errors are 46 (a), 68 (b), 66 (c) and $21 \mathrm{~m}$ (d) 


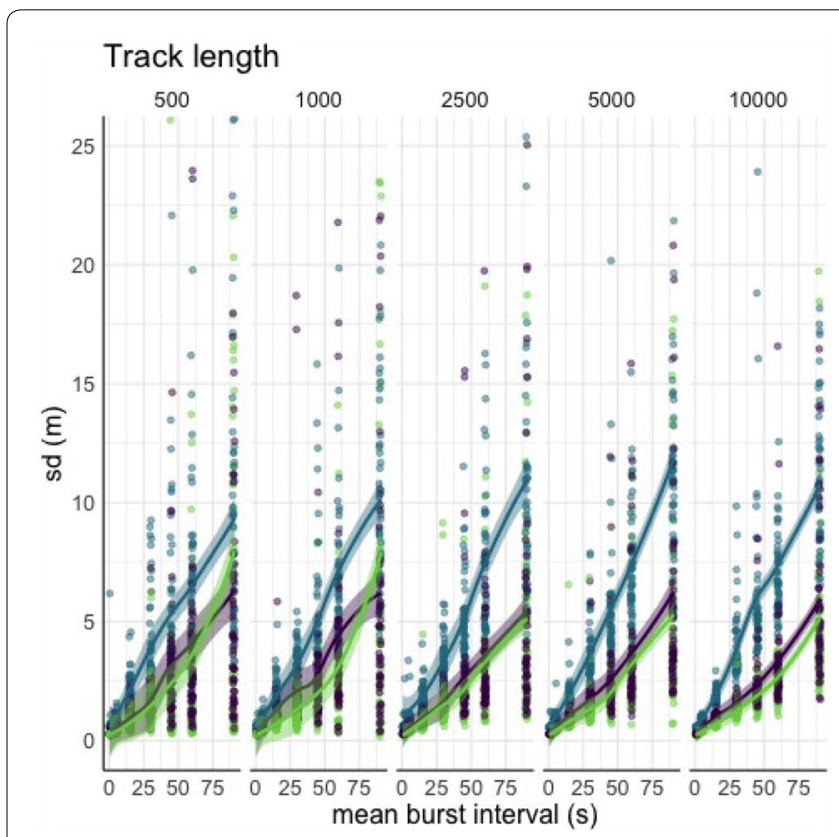

a

\section{Out-of-array shifts}

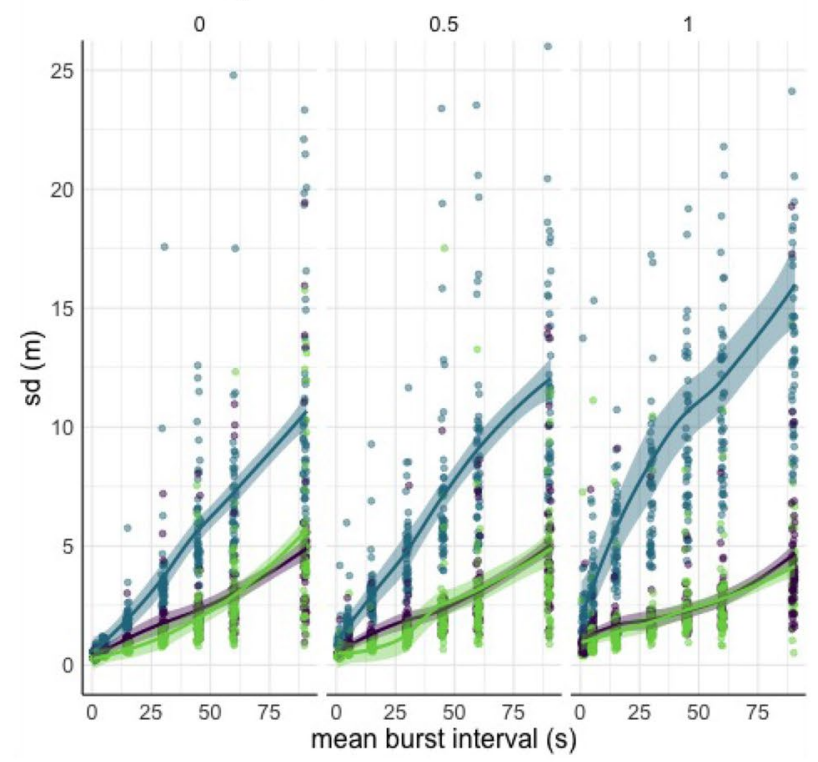

c

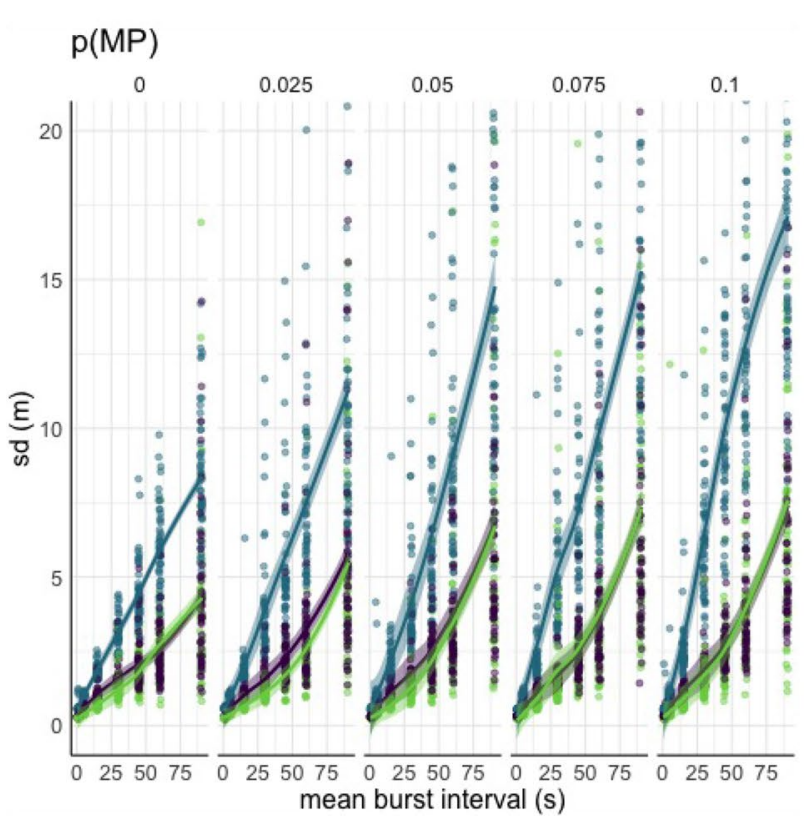

b

Temporal receiver resolution (1E-6 s)

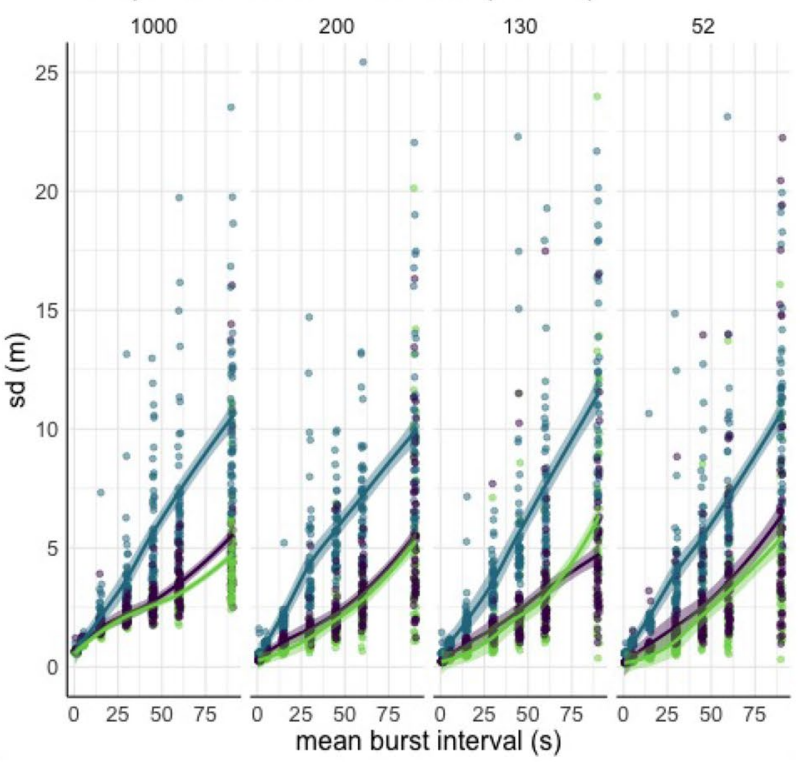

d

Fig. 2 YAPS performance in different settings. The four plots show the standard deviation on the error resulting from the combined effect of burst interval properties with a varying track lengths, $\mathbf{b}$ probabilities of multipath $\mathrm{p}(\mathrm{MP})$, c shifts out-of-array and $\mathbf{d}$ temporal receiver resolution. Each panel shows the results for random burst interval (blue), known random burst interval (purple) and stable burst interval (green) and for different mean burst intervals (x-axis). Each dot represents standard deviation on the error of one track. Each combination of settings was run on 50 tracks. When not under variation, track length, p(MP), shift and resolution were respectively fixed at 5000, 2.5\%, 0 and $200 \mu$ s. The line on top of the data is a LOcally Estimated Scatterplot Smoothing (LOESS) smoother with 95\%-confidence interval showing respectively the general pattern (i.e. average value per setting) and the uncertainty on the smoother. To enhance the visualisation, standard deviations above $25 \mathrm{~m}$ are not shown. The maximum occurring standard deviations are 62 a, 62 b, 79 c and 35 m d 
errors is large, extending from 0.2 to $72 \mathrm{~m}$ for tracks of $500 \mathrm{~s}$ with 60 to $90 \mathrm{~s}$ mean burst interval. Tracks longer than $2500 \mathrm{~s}$ only gain by increasing track length if the burst interval is random. Note that tracks of $500 \mathrm{~s}$ with 45,60 or $90 \mathrm{~s}$ mean burst interval contain only about 11 , 8 or 6 observations, respectively. For small burst intervals (up to $15 \mathrm{~s}$, corresponding to at least 33 observations when the track length is $500 \mathrm{~s}$ ), the error barely changes with track length, hence short tracks are no issue. For larger stable or known random burst intervals (30 s and more), about 40 observations are needed (e.g. $60 \mathrm{~s}$ burst interval when track length is $2500 \mathrm{~s}$ ) to minimise the error. Three tracks with only six observations could not be estimated by YAPS (at least not within 100 retries). Remarkably, for tracks of $10,000 \mathrm{~s}$ with random burst interval, some large errors still occur despite the smaller median error. This is also confirmed when looking at Fig. 2a: the distribution of standard deviations on each track is similar for each track length. This indicates that large errors still occur for some tracks, even if the number of observations is high.

\section{Effect of reflectivity}

Multipath probabilities up to 5\% have a minor effect on the performance of YAPS (Fig. 1b). Higher probabilities cause an increase in errors, but only for large burst intervals: the errors are about twice as large for $10 \%$ versus 5\% (or no) reflectivity, and this for all burst types. Regarding the standard deviation on the errors, even a small increase in multipath probability does have an effect (Fig. 2b), meaning that the spread on positioning errors for a track increases rapidly, and this for all burst types. Small burst intervals up to $30 \mathrm{~s}$ are barely affected by increasing reflections (Fig. 1b). When the random sequence is known, the average of the median errors does not exceed $2.5 \mathrm{~m}$ in worst-case scenario (i.e. $10 \%$ reflections and largest burst interval), whereas a purely random burst interval then results in median errors of $10 \mathrm{~m}$ on average, and even up to $49 \mathrm{~m}$.

\section{Effect of out-of-array}

For each burst type and all burst intervals, the error is about twice as large for tracks out-of-array versus tracks completely inside the array (Fig. 1c). However, since the error on random burst interval tracks is already large (especially for large burst intervals), the effect of doubling the error is worse, resulting in median errors of $14 \mathrm{~m}$ on average. Despite larger errors out-of-array, the form of the track is still captured quite well, even for random burst interval (Fig. 3). When a track is half out-of-array, the error increases only slightly. The standard deviation on the error is larger for tracks out-of-array than inside or half out-of-array, in the case of random burst intervals
(Fig. 2c). For stable and known random intervals, there is no increase in the standard deviation for out-of-array positioning, despite the increase in median errors. This indicates a general upwards shift in all errors.

\section{Effect of temporal receiver resolution}

Improvement of the temporal receiver resolution affects the average error on al burst intervals when the burst type is stable or the random sequence is known (Fig. 1d). However, for random burst intervals, resolutions better than $200 \mu$ s do not improve the track estimation further. Whilst the error usually increases with increasing burst interval, for stable burst intervals this effect practically disappears with a resolution better than $200 \mu$ s. Standard deviations on the error show the same patterns for different receiver resolutions (Fig. 2d). Apparently, temporal receiver resolution has only an effect on the magnitude and not on the spread of the error. This is logic, since it only affects how accurately the receiver is measuring and does not interfere with other processes that influence the performance of the positioning algorithm.

\section{Discussion}

The parameter that mostly affects YAPS' positioning accuracy is the burst type. When the next time of transmission is known (or at least follows a Gaussian distribution), the estimation of transmission time is quite simple and informed by the surrounding transmission times. If this information is missing, the independent estimation of each time of transmission adds uncertainty and inaccuracies to the model. A stable burst interval hence results almost always in better accuracy, and quite large burst intervals (up to $60 \mathrm{~s}$ ) can be chosen without really hampering the median accuracy on a track (although individual points might have higher errors). When using stable burst interval transmitters, there is a risk that transmitters are pinging in phase and hence colliding constantly, making decoding impossible. To avoid this issue, random burst intervals can be used. In this case, the availability of the random sequence significantly improves the results of YAPS estimations, actually approaching the accuracy reached with stable burst interval.

The choice of burst interval has a small influence on median error when the burst interval is stable or when the random burst interval sequence is known and when conditions are good (i.e. enough observations, a resolution better than $1 \mathrm{~ms}$ and reflectivity lower than $10 \%$ ). In contrast, the error on random burst interval tracks increases rapidly with increasing burst interval. Even if the median error is not affected, the standard deviation per track does increase with burst interval in all situations, hence positions with larger error might be expected when the burst interval is large. Note that large 


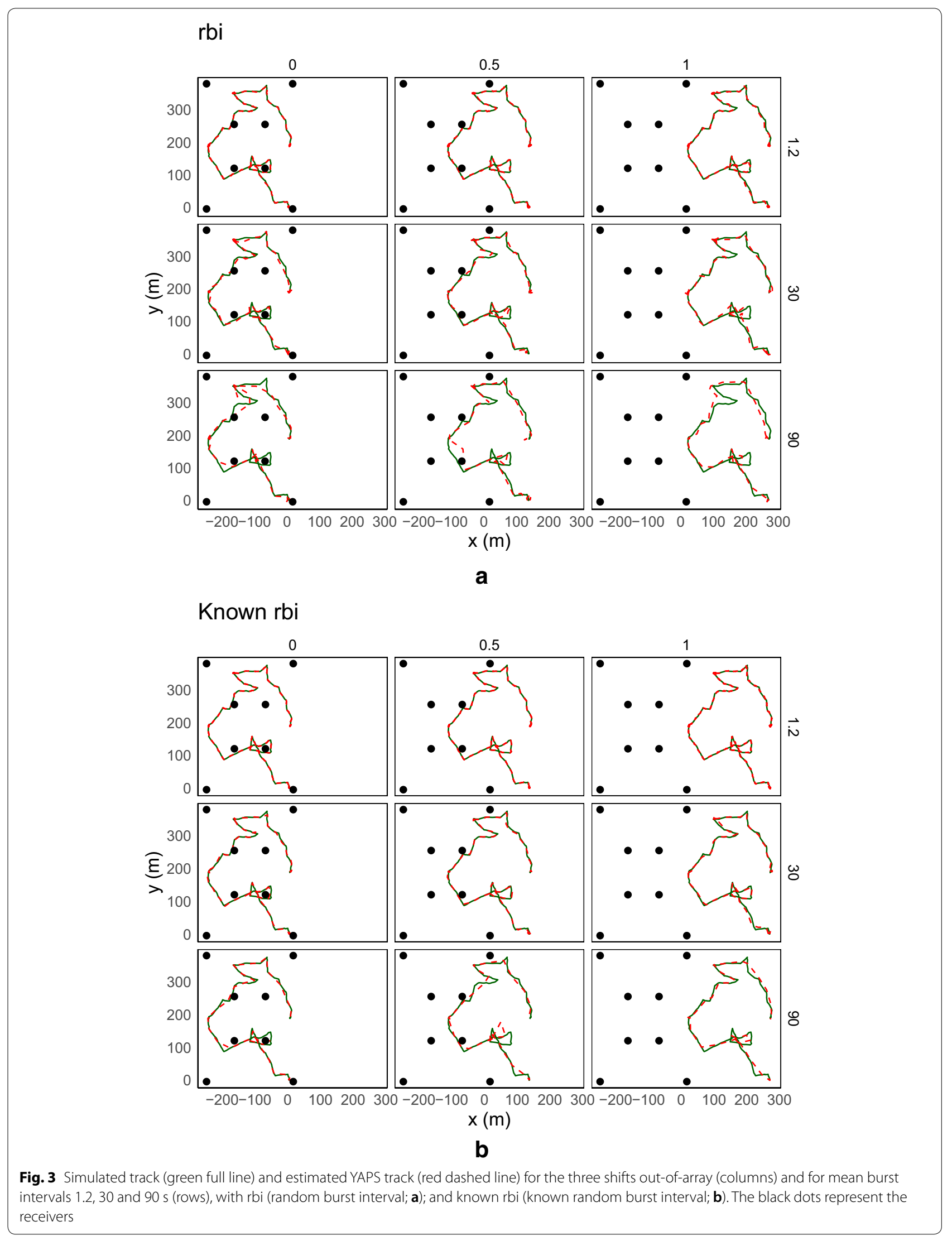


burst intervals inherently come with information loss: between subsequent positions, the best estimation of an animal's behaviour is a straight line. On the other hand, very small burst intervals (around $1 \mathrm{~s}$ ) require high temporal receiver resolution and result in excessively large datasets, challenging the processing limits of most personal computers and therefore hampering data handling and analysis.

In some cases, YAPS might fail to converge, which happened for three tracks of six observations in this simulation study. When a fish passes quickly through an array, and the burst interval of its transmitter is large, very few observations of its transmitter may be available. This provides very little information to fit the movement model and might result in a wide range of errors, from very small to very large. If the burst type is random, each time of transmission must be estimated separately, which additionally complicates the task with only about 10 TOA-data available. For small burst intervals, about 30 observations are sufficient to avoid effects of too few data. For large burst intervals, it depends on the burst type: 40 observations are enough when the burst interval is stable or when the random burst interval sequence is known. For large random burst intervals, more observations are beneficial, but accuracy is never completely guaranteed: even at 10,000 s track length, some tracks are estimated with high error. Hence, small burst intervals are advised when high accuracy is required.

Comparing different levels of reflection revealed that YAPS is very robust against errors due to reflections when the burst interval is small enough (i.e. not larger than $30 \mathrm{~s}$ ), even up to $10 \%$ reflectivity. This robustness can be attributed to the use of the movement model, keeping positions within biologically plausible limits. When the burst interval is large and random, it is more difficult to intercept reflected signals, since the time of the next transmission is not known and has to be estimated independently. However, this effect is only significant for multipath probabilities larger than 5\%. Moreover, if the random sequence is available, the average of median track errors stays limited to $2.5 \mathrm{~m}$ even for the largest burst interval. This shows the benefit and importance of knowing the random sequence.

The accuracy of YAPS is not much affected when only half of the track is outside of the array. However, tracks completely outside the array are only half as accurate, but still capture the (simulated) behaviour (Fig. 3). This can be explained as follows: since YAPS uses Time Of Arrival directly, instead of Time Difference Of Arrival, it is geometrically based on intersecting circles instead of hyperbolas. As is the case for hyperbolas [13], the intersection of circles is more sensitive to errors in measured arrival time when the transmitter is outside the receiver array than when it is inside. The direct use of TOA (and hence circles) is only possible because YAPS iteratively estimates the time of transmission and uses all TOA information simultaneously. The latter helps in avoiding completely erroneous positions once the transmitter is leaving the array contour, hence better preserving the behaviour. Although the main zone of interest is usually within the limits of the receiver array, sometimes it might be interesting to obtain information on how a fish is arriving at the study site. This information is mostly missing in TDOA studies.

Regardless of the positioning algorithm, fine-scale positioning with high accuracy requires receivers with a sub-millisecond temporal resolution. The temporal receiver resolution depends on technological choices of the manufacturer and determines how accurate a positioning system can physically be. This simulation study allowed to quantify the combined effect of resolution and other parameters. For instance, a PPM system, that has a temporal resolution of $1 \mathrm{~ms}$ and typically an average burst interval not lower than $30 \mathrm{~s}$, has a minimal error of about $2 \mathrm{~m}$ in best-case scenario. In contrast, a CDMA system with $52 \mu$ s resolution, can be $20 \mathrm{~cm}$ accurate for the same burst interval.

\section{Conclusions}

This study aimed to inform users and manufacturers on potential areas of improvement. As it turned out, the main point of improvement is the choice of burst type, which is of major importance for the performance of YAPS. Whereas the use of stable burst intervals leads in nearly all cases to better results, the poor performance on random burst interval data can be significantly improved when the random sequence is available. Therefore, we strongly urge manufacturers to store this information and make it available to customers. Another potential area of improvement is the temporal receiver resolution, which in turn determines the minimal accuracy that can be reached. Resolutions better than $1 \mathrm{~ms}$ are recommended for accurate fine-scale positioning.

In addition, the study aimed to help users in the choice of system settings. The choice of burst interval depends on many factors, such as the scale of the study, the research questions and hence the required accuracy. Whenever possible, small burst intervals are beneficial for the accuracy when low numbers of observations can be expected (i.e. fast-moving animals), in reflective environments and when there is interest in information from outside the receiver array. However, the benefit of small burst intervals is especially high for (unknown) random burst intervals. Very small burst intervals (around $1 \mathrm{~s}$ ) 
result in excessively large datasets, needlessly complicating the data analysis.

As the design of the receiver array is often constraint to the specific lay-out of a study area, we conducted this simulation study on a fixed array design. However, a study that focusses on comparing array designs of different quality, geometry, spacing and number of receivers, would be an interesting follow-up to test the robustness of the YAPS algorithm.

\section{Methods}

Below we first introduce the studied parameters ("Studied parameters" section) and then explain how their effects on the positioning error were investigated in the simulation study ("Set-up of the simulation study" section).

\section{Studied parameters}

\section{Signal characteristics: burst type and interval}

In case of a stable burst interval (sbi), the YAPS algorithm can easily predict the time of the next transmission. In reality, the interval between transmission is not exactly constant, because the transmitter's internal clock is imprecise and prone to drifting (personal observation of the authors). Hence, the burst interval $t(i)-t(i-1)$ can be approximated by a random walk or Gaussian distribution with standard deviation $\sigma$ [15]:

$$
t(i)-t(i-1) \mid t(i-1), t(i-2) \sim N\left(t(i-1)-t(i-2), \sigma^{2}\right) .
$$

When the random sequence is unknown to the user, it can only be modelled as a random interval. In the YAPS algorithm, the random burst interval is approximated by a uniform distribution between minimum and maximum burst interval $(a, b)$, and is estimated for each ping independently, resulting in the following model for burst interval:

$$
t(i)-t(i-1) \mid t(i-1) \sim U(a, b) .
$$

In contrast, if the random sequence $S$ is known, YAPS can utilise this information to predict the time of next transmission, whilst incorporating deviation caused by drift of the internal transmitter clock. The sequence of known random transmission times $t_{\mathrm{rbi}}$ can be expressed as:

$$
t_{\mathrm{rbi}}(i)=t_{\mathrm{rbi}}(i-1)+S(i-1)
$$

and the burst interval can be modelled as:

$$
\begin{aligned}
& t_{\mathrm{rbi}}(i)-t_{\mathrm{rbi}}(i-1) \mid t_{\mathrm{rbi}}(i-1), t_{\mathrm{rbi}}(i-2) \\
& \sim N\left(t_{r b i}(i-1)-t_{\mathrm{rbi}}(i-2), \sigma^{2}\right) .
\end{aligned}
$$

In this study, we varied the average burst interval between 1.2 and $120 \mathrm{~s}$ (Table 1 ).
Table 1 Stable burst intervals (sbi) and corresponding minimum and maximum random burst intervals (rbi)

\begin{tabular}{lll}
\hline $\mathbf{s b i}(\mathbf{s})$ & Min rbi (s) & Max rbi (s) \\
\hline 1.2 & 1.1 & 1.3 \\
5 & 3 & 7 \\
15 & 10 & 20 \\
30 & 20 & 40 \\
45 & 30 & 60 \\
60 & 40 & 80 \\
90 & 60 & 120 \\
\hline
\end{tabular}

Each stable burst interval is also the average of the corresponding random burst interval limits

\section{Number of observations or track length}

Point-by-point positioning methods such as TDOA can calculate a position no matter the number of transmissions, as long as the signal is detected by at least three receivers. A fish with a large burst interval-transmitter, swimming fast through a receiver array, can result in one single TDOA position. In the YAPS algorithm, a minimum amount of data is required to fit the movement model to the TOA data. We verified the YAPS performance on tracks with a duration of 500, 1000, 2500, 5000 and $10,000 \mathrm{~s}$. Note that the number of calculated positions depends on the burst interval, for instance, a track of $500 \mathrm{~s}$ with a 90-s mean burst interval contains on average 6 positions. In this paper, we use 'track length' to refer to the duration of the track, whilst 'number of observations' indicate how many observations can possibly result from a track length with given burst interval.

\section{Reflectivity of the environment}

The reflectivity of the environment is expressed as the probability on a multipath signal $\mathrm{p}(\mathrm{MP})$. Reflection of a signal leads to an increase in path length between transmitter and receiver, resulting in an offset of the arrival time compared to normal arrival time (i.e. without reflections). Hence, we introduced multipath in the simulation study by offsetting a fraction of the TOA-data with a random value between 0.035 and $0.209 \mathrm{~s}$, which corresponds to an error of 50 to $300 \mathrm{~m}$ (assuming $1435 \mathrm{~m} / \mathrm{s}$ sound speed in water). These values, that are typically within the detection range of a receiver, are representative for outliers in TOA-data in different kinds of environments (personal experience of the authors). We varied $\mathrm{p}(\mathrm{MP})$ between 0 and $10 \%$ by $2.5 \%$ steps.

\section{Out-of-array positioning}

We verified the accuracy of YAPS in estimating out-ofarray tracks by comparing tracks within the array, with the results of shifting tracks a half array-length out of 
Table 2 Temporal receiver resolutions for different systems and providers, which were used in the simulation study

\begin{tabular}{ll}
\hline System & $\begin{array}{l}\text { Temporal } \\
\text { resolution }\end{array}$ \\
\hline Vemco PPM & $1 \mathrm{~ms}$ \\
Thelma Biotel PPM & $1 \mathrm{~ms}$ \\
Lotek $76 \mathrm{kHz}$ & $200 \mu \mathrm{s}$ \\
Vemco HR & $130 \mu \mathrm{s}$ \\
Lotek $200 \mathrm{kHz}$ & $52 \mu \mathrm{s}$ \\
\hline
\end{tabular}

the array (shift $=0.5)$, and completely out of the array $($ shift $=1)$.

\section{Temporal receiver resolution}

The temporal receiver resolution determines the accuracy that is physically achievable, for instance, $1 \mathrm{~ms}$ resolution corresponds to $1.4 \mathrm{~m}$ accuracy (assuming $1435 \mathrm{~m} / \mathrm{s}$ sound speed in water). We compared YAPS performance on different temporal resolutions, corresponding to systems of the Vemco, Thelma Biotel and Lotek company (Table 2).

\section{Set-up of the simulation study}

To verify the influence of number of observations, we simulated 50 tracks for each 5 track lengths, in an array of 8 equally spread receivers (Fig. 4). The array consisted of 2 concentric rectangles, with receivers spaced 20 to $30 \mathrm{~m}$ apart, providing a basic but robust design for positioning. The simulated tracks were based on a correlated random walk movement model, with alternating periods of low and high movement. For each track, we also simulated sound speed data, following a random walk model.

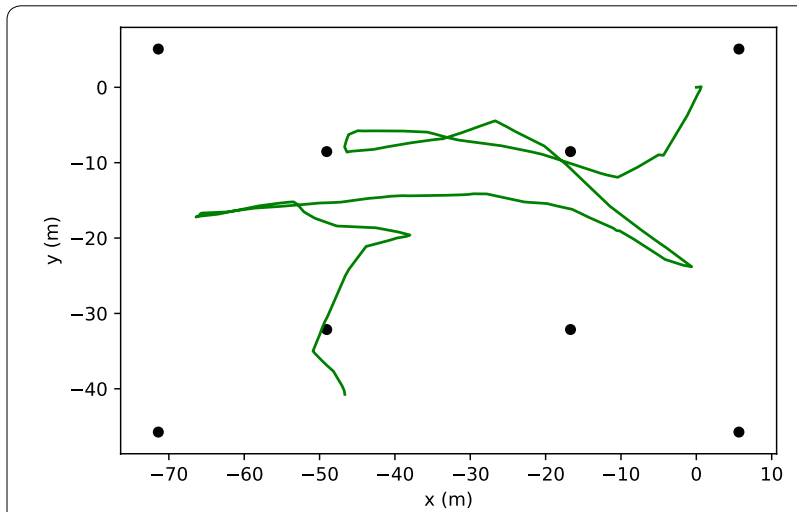

Fig. 4 Example of a simulated track of $1000 \mathrm{~s}$ (green) and corresponding receiver array (black dots)

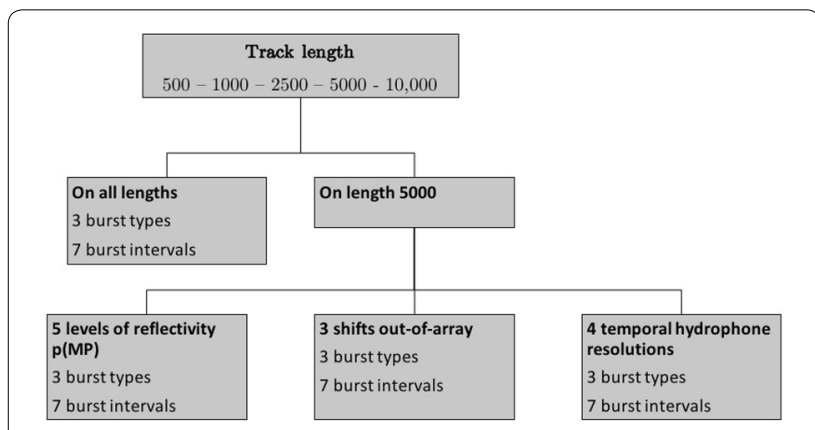

Fig. 5 Graphic showing the simulation outline

We assumed a constant sound speed and homogeneous sound speed profile. For each of the resulting 250 tracks $(50 * 5)$, we simulated TOA data for the 7 burst intervals (Table 1) and 3 burst types. To verify the effect of other parameters, we used only the 50 tracks of track length 5000. On these tracks, we simulated TOA data for the 7 burst intervals and 3 burst types, combined with the 5 different probabilities of multipath $\mathrm{p}(\mathrm{MP})$ and 4 different temporal receiver resolutions, giving in total 189 settings $(21 * 5+21 * 4)$. We subjected the 50 tracks with length 5000 to 3 shifts relative to the array: no shift, half-out and completely out of array. For these 150 tracks, we again simulated TOA data for 7 burst intervals and 3 burst types (21 settings). Figure 5 summarises the simulation outline and the used R code can be found on-line [28].

All TOA data were calculated with $60 \%$ probability of missing detections, i.e. on average 3 (out of 8 ) receivers detecting the signal. The parameters $\mathrm{p}(\mathrm{MP})$ and temporal receiver resolution were fixed at $2.5 \%$ and $200 \mu \mathrm{s}$ respectively, when not varied. Finally, we ran the YAPS algorithm on each of the 17,850 TOA-datasets $(250 * 21+$ $50 * 189+150 * 21$ ) to produce estimated tracks, allowing 100 attempts to converge. For visualisation, we plotted an LOESS smoother on top of the points representing the median error on each estimated track. This line and its $95 \%$-confidence interval show respectively the general pattern and the uncertainty on the smoother. The error on each position is calculated as the distance between simulated and estimated position.

\footnotetext{
Abbreviations

p(MP): Probability of multipath; $\sigma$ : Standard deviation of Gaussian distribution; $S$ : Random sequence; $t_{\text {rbi: }}$ Known random transmission time; $t(i)-t(i-1)$ : Burst interval; BPSK: Binary Phase Shift Key; CDMA: Code Division Multiple Access; LOESS: LOcally Estimated Scatterplot Smoothing; GPS: Global Positioning System; HPE: Horizontal Positioning Error; HR: High Residency; PPM: Pulse Position Modulation; RMSE: Root Mean Square Error; sd: Standard deviation; sbi: Stable burst interval; rbi: Random burst interval; TDOA: Time Difference Of Arrival; TOA: Time Of Arrival; VPS: Vemco Positioning System; YAPS: Yet Another Positioning System.
} 


\section{Acknowledgements}

This work was a collaboration between Ghent University, the Research Institute for Nature and Forest (INBO) and the Technical University of Denmark.

\section{Authors' contributions}

$\mathrm{J}$ interpreted the data and wrote the manuscript, HB wrote the code for the simulations, delivered the data and contributed to the interpretation. IP, TDM AM and IN supervised the work of JV. All authors read and approved the final manuscript.

\section{Funding}

J. Vergeynst is a Ph.D. fellow funded by the Special Research Fund (BOF) of Ghent University.

\section{Availability of data and materials}

The R code to run the simulation study is available on GitHub: https://githu b.com/JennaVergeynst/YAPS_simulation_study. Archived as: Vergeynst, Jenna, and Baktoft, Henrik. (2019, November 25). JennaVergeynst/YAPS_simulation_study:YAPS simulation study. Zenodo. http://doi.org/10.5281/zenod 0.3552440

\section{Ethics approval and consent to participate}

The study is approved by the Ethical Committee of the Research Institute for Nature and Forest (ECINBO09).

\section{Consent for publication}

Not applicable.

\section{Competing interests}

The authors declare that they have no competing interests.

\section{Author details}

${ }^{1}$ Department of Data Analysis and Mathematical Modelling, Ghent University, Coupure Links 653, 9000 Ghent, Belgium. ${ }^{2}$ National Institute of Aquatic Resources, Technical University of Denmark, Vejls vej 39, 8600 Silkeborg, Denmark. ${ }^{3}$ Research Institute for Nature and Forest (INBO), Havenlaan 88/73, 1000 Brussels, Belgium. ${ }^{4}$ Department of Civil Engineering, Ghent University, Sint-Pietersnieuwstraat 41, 9000 Ghent, Belgium.

Received: 2 December 2019 Accepted: 25 June 2020 Published online: 16 July 2020

\section{References}

1. Henderson HF, Hasler AD, Chipman GG. An ultrasonic transmitter for use in studies of movements of fishes. Trans Am Fish Soc. 1966;95(4):350-65. https://doi.org/10.1577/1548-8659(1966)95[350:AUTFUI]2.0.CO;2.

2. Cochran WW, Lord RD. A radio-tracking system for wild animals. J Wildl Manage. 1963;27(1):9-24. https://doi.org/10.2307/3797775.

3. LeMunyan CD, White W, Nyberg E, Christian JJ. Design of a miniature radio transmitter for use in animal studies. J Wild Manage. 1959;23(1):107-10. https://doi.org/10.2307/3797755.

4. Trefethen PS. Sonic equipment for tracking individual fish. U.S. fish and wildlife service special science report. Fisheries. 1956;179:1-11.

5. DeCelles G, Zemeckis D. Acoustic and radio telemetry. In: Cadrin SX, Kerr LA, Mariani S, editors. Stock identification methods. Cambridge: Academic Press; 2014. p. 397-428. https://doi.org/10.1016/B978-0-12-39700 3-9.00017-5.

6. Ward MP, Sperry JH, Weatherhead PJ. Evaluation of automated radio telemetry for quantifying movements and home ranges of snakes. J Herpetol. 2013;47(2):337-45. https://doi.org/10.1670/12-018.

7. Harbicht AB, Castro-Santos T, Ardren WR, Gorsky D, Fraser DJ. Novel, continuous monitoring of fine-scale movement using fixed-position radiotelemetry arrays and random forest location fingerprinting. Methods Ecol Evol. 2017;8:850-9. https://doi.org/10.1111/2041-210X.12745.

8. Hussey NE, Kessel ST, Aarestrup K, Cooke SJ, Cowley PD, Fisk AT, Harcourt RG, Holland KN, Iverson SJ, Kocik JF, Flemming JEM, Whoriskey FG. Aquatic animal telemetry: a panoramic window into the underwater world. Science. 2015;348(6240):1255642. https://doi.org/10.1126/scien ce. 1255642 .

9. Vergeynst J, Pauwels I, Baeyens R, Coeck J, Nopens I, De Mulder T, Mouton A. The impact of intermediate-head navigation locks on downstream fish passage. River Res Appl. 2019;35:224-35. https://doi.org/10.1002/rra.3403.

10. Verhelst P, Buysse D, Reubens J, Pauwels I, Aelterman B, Van Hoey S, Goethals P, Coeck J, Moens T, Mouton A. Downstream migration of European eel (Anguilla anguilla L.) in an anthropogenically regulated freshwater system: implications for management. Fish Res. 2018;199:252-62. https:// doi.org/10.1016/j.fishres.2017.10.018.

11. Aarestrup K, Baktoft H, Thorstad EB, Svendsen JC, Höjesjö J, Koed A. Survival and progression rates of anadromous brown trout kelts Salmo trutta during downstream migration in freshwater and at sea. Mar Ecol Prog Ser. 2015;535:185-95. https://doi.org/10.3354/meps11407.

12. Aarestrup K, Baktoft H, Koed A, Del Villar-Guerra D, Thorstad EB. Comparison of the riverine and early marine migration behaviour and survival of wild and hatchery-reared sea trout Salmo trutta smolts. Mar Ecol Prog Ser 2014;496:197-206. https://doi.org/10.3354/meps10614.

13. Smith, F. Understanding HPE in the VEMCO positioning system (VPS). Technical report 2013. http://vemco.com/wp-content/uploads/2013/09/ understanding-hpe-vps.pdf.

14. Vergeynst J, Vanwyck T, Baeyens R, De Mulder T, Nopens I, Mouton A, Pauwels I. Acoustic positioning in a reflective environment: going beyond point-by-point algorithms. Anim Biotelem. 2020;8:1-17.

15. Baktoft $H$, Gjelland $K \varnothing, \varnothing k$ land $F$, Thygesen UH. Positioning of aquatic animals based on time-of-arrival and random walk models using YAPS (Yet Another Positioning Solver ). Sci Rep. 2017;7:1-10. https://doi. org/10.1038/s41598-017-14278-z.

16. Binder TR, Holbrook CM, Hayden TA, Krueger CC. Spatial and temporal variation in positioning probability of acoustic telemetry arrays: fine-scale variability and complex interactions. Anim Biotelem. 2016;4(1):1-15. https ://doi.org/10.1186/s40317-016-0097-4.

17. Vemco: How do VEMCO coded tags work. 2019. https://support.vemco .com/s/article/How-do-VEMCO-coded-tags-work.

18. Sacko D, Kéita AA. Techniques of modulation: pulse amplitude modulation, pulse width modulation, pulse position modulation. Int J Eng Adv Technol. 2017;7(2):100-8.

19. Simpfendorfer CA, Heupel MR, Collins AB. Variation in the performance of acoustic receivers and its implication for positioning algorithms in a riverine setting. Can J Fish Aquat Sci. 2008;65(3):482-92. https://doi. org/10.1139/f07-180.

20. Guzzo MM, Van Leeuwen TE, Hollins J, Koeck B, Newton M, Webber DM, Smith FI, Bailey DM, Killen SS. Field testing a novel high residence positioning system for monitoring the fine-scale movements of aquatic organisms. Methods Ecol Evol. 2018;9:1478-88. https://doi. org/10.1111/2041-210X.12993.

21. Leander J, Klaminder J, Jonsson M, Brodin T, Leonardsson K, Hellström G. The old and the new: evaluating performance of acoustic telemetry systems in tracking migrating Atlantic salmon (Salmo salar) smolt and European eel (Anguilla anguilla) around hydropower facilities. Can J Fish Aquat Sci. 2019;77:177-87.

22. Niezgoda G, Benfield M, Sisak M, Anson P. Tracking acoustic transmitters by code division multiple access (CDMA)-based telemetry. Hydrobiologia. 2002;483:275-86. https://doi.org/10.1023/A:1021368720967.

23. Cooke SJ, Niezgoda GH, Hanson KC, Suski CD, Phelan FJS, Tinline R, Philipp DP. Use of CDMA acoustic telemetry to document 3-D positions of fish: Relevance to the design and monitoring of aquatic protected areas. Mar Technol Soc J. 2005;39(1):31-41. https://doi.org/10.4031/0025332057 87521659.

24. Baktoft H, Gjelland KØ, Økland F, Rehage JS, Rodemann JR, Corujo RS, Viadero N, Thygesen UH. Opening the black box of high resolution fish tracking using yaps. bioRxiv, the prepint server for biology. 2019. pp. $1-14$.

25. Vergeynst J. JennaVergeynst/time_synchronization: code for time synchronisation of an acoustic positioning system. Zenodo. 2019. https://doi. org/10.5281/zenodo.3540681.

26. Pincock DG. Understanding the performance of VEMCO $69 \mathrm{kHz}$ single frequency acoustic telemetry. Technical report 2008. 
27. Holmes N, Browne A, Montague C. Acoustic properties of concrete panels with crumb rubber as a fine aggregate replacement. Constr Build Mater. 2014;73:195-204. https://doi.org/10.1016/j.conbuildma t.2014.09.107.

28. Vergeynst J, Baktoft H. JennaVergeynst/YAPS_simulation_study: YAPS simulation study. Zenodo. 2019. https://doi.org/10.5281/zenodo.3552440.

\section{Publisher's Note}

Springer Nature remains neutral with regard to jurisdictional claims in published maps and institutional affiliations.
Ready to submit your research? Choose BMC and benefit from:

- fast, convenient online submission

- thorough peer review by experienced researchers in your field

- rapid publication on acceptance

- support for research data, including large and complex data types

- gold Open Access which fosters wider collaboration and increased citations

- maximum visibility for your research: over $100 \mathrm{M}$ website views per year

At $\mathrm{BMC}$, research is always in progress.

Learn more biomedcentral.com/submissions 